\title{
Neuroimaging Features, Follow-Up Analyses, and Comparisons Between Asymptomatic and Symptomatic Neurosyphilis
}

\author{
Xian-Jin Shang $\cdot$ Cai-Feng He $\cdot$ Biao Tang $\cdot$ Xiao-Li Chang • \\ Chao Ci · Hong Sang (D)
}

Received: December 16, 2019 / Published online: March 2, 2020

(C) The Author(s) 2020

\begin{abstract}
Introduction: Many studies have explored the imaging characteristics of patients with neurosyphilis, but no systematic study has been made on the neuroimaging changes after antisyphilitic treatment. The purpose of this study was to examine neuroimaging differences before and after treatment, comparing patients with asymptomatic and symptomatic neurosyphilis.
\end{abstract}

Enhanced Digital Features To view enhanced digital features for this article go to https://doi.org/10.6084/ m9.figshare.11841522.

Electronic supplementary material The online version of this article (https://doi.org/10.1007/s13555020-00361-3) contains supplementary material, which is available to authorized users.

\section{X.-J. Shang}

Department of Neurology, Yijishan Hospital of Wannan Medical College, Wuhu 241001, People's Republic of China

C.-F. He · H. Sang $(\bowtie)$

Jinling Hospital Department of Dermatology,

Nanjing Medical University, Nanjing 210002,

People's Republic of China

e-mail: sanghong@nju.edu.cn

C.-F. He · B. Tang · X.-L. Chang · C. Ci

Department of Dermatology, Yijishan Hospital of

Wannan Medical College, Wuhu 241001, People's

Republic of China
Methods: A total of 102 patients with neurosyphilis, including 60 cases of symptomatic neurosyphilis and 42 cases of asymptomatic neurosyphilis, were identified between December 2012 and June 2019. Their demographics, medical histories, serological tests of peripheral blood and cerebrospinal fluid, and especially neuroimaging features before and after antisyphilitic treatment were collected and analyzed.

Results: The patients presented with variable clinical and neuroimaging features, including cerebral infarction or hemorrhage, atrophy, demyelination, arteritis, encephalitis, and hippocampal sclerosis. A total of 29 neuroradiological re-examinations were performed in 19 patients treated with anti-syphilitic medicine. The results indicated that some patients still presented neuroradiological progression after treatment, including $42.1 \%$ showing infarction lesions, $47.4 \%$ mild to severe brain atrophy, and $15.8 \%$ white matter demyelination.

Conclusion: The clinical and neuroimaging features of neurosyphilis patients are diverse, and their follow-up neuroimaging continued to show progression even with standardized treatment.

Keywords: Anti-syphilitic treatment; Follow up; Neuroimaging; Neurosyphilis; Treponema pallidum 


\section{Key Summary Points}

Neuroimaging examinations in patients with neurosyphilis are important for the differential clinical diagnosis, and the presentations are diverse. However, no systematic study has investigated neuroimaging changes or progression after anti-syphilitic treatment.

This study will answer whether neuroimaging and symptomology show consistent improvement if patients with neurosyphilis are treated with standardized anti-syphilitic medicine.

Patients with neurosyphilis presented with variable clinical and neuroimaging features, including cerebral infarction or hemorrhage, atrophy, demyelination, arteritis, encephalitis, and hippocampal sclerosis.

And the follow-up neuroimaging demonstrated that some patients still presented neuroradiological progression after standardized anti-syphilitic treatment, including $42.1 \%$ showing infarction lesions, $47.4 \%$ mild to severe brain atrophy, and $15.8 \%$ white matter demyelination.

Neurosyphilis patients show neuroradiological progression after antisyphilitic treatment, even if the patients do not have any clinical symptoms, suggesting that we need to continue imaging follow-up and treatment of these patients.

\section{INTRODUCTION}

Neurosyphilis (NS) is a syphilitic infection of the central nervous system (CNS) caused by the organism Treponema pallidum, which triggers subacute or chronic inflammation in mesenchymal and parenchymal tissues, finally causing a spectrum of disease processes with different neurological manifestations. Patients with syphilis are often infected by sexual contact or other contact with the body fluids of an infected person, and the pathogenic bacteria then multiply in the body and gradually invade the CNS. The condition can be divided into early and late NS, depending on the clinical features and duration from initial infection [1]. Alternatively, according to the scope of the brain tissue affected, patients can also be dichotomized into interstitial NS or parenchymatous NS, which can have the forms of meningitis, meningovascular NS, general paresis, tabes dorsalis, and gummatous NS. There is no clear boundary between the different forms, with there being a substantial overlap in clinical symptoms and signs [2, 3]. According to the guidelines, patients diagnosed with NS need prompt treatment with high-dose intravenous penicillin [4]. However, most patients with NS present with nonspecific symptoms, so the rates of clinical misdiagnosis and missed diagnosis are relatively high [5]. Currently, the diagnostic criteria for NS are based on a comprehensive assessment of clinical characteristics, cerebrospinal fluid (CSF) pleocytosis and protein levels, and syphilis serological testing of the peripheral blood and CSF.

Neuroimaging examinations in patients with NS are important for the differential clinical diagnosis and follow-up, with the presentations being diverse [6]. Although several studies have analyzed clinical and laboratory characteristics of asymptomatic and symptomatic neurosyphilis patients, to the best of our knowledge, no systematic study has investigated neuroimaging changes after anti-syphilitic treatment [7-10]. Herein, we retrospectively reviewed 102 patients with NS who were examined at our center and made comparisons between asymptomatic and symptomatic NS patients. We also evaluated the initial and follow-up imaging characteristics in some patients. 


\section{METHODS}

\section{Study Design}

This is a retrospective observational study of inpatients diagnosed with NS at our hospital between December 2012 and June 2019. Eligible patients were selected from the hospital information system and their medical history, coexisting conditions, neurological examinations, peripheral blood and CSF laboratory testing, and initial and follow-up neuroimaging were recorded and evaluated. The study protocol was approved by Wannan Medical College (IRB: 201201) with a waiver for informed consent from participants because of its retrospective nature.

\section{Study Patients}

All study participants were 18 years old or older, were diagnosed with HIV-negative neurosyphilis, and met all the following conditions: (1) Treponema pallidum particle agglutination assay (TPPA) and toluidine red unheated serum test (TRUST) titer were tested in serum and the serum TPPA was positive; (2) the serologic test was combined with TRUST positivity in CSF or a CSF white blood cell count of $>5$ cells/ $\mu$ l or CSF protein of $>45 \mathrm{mg} / \mathrm{dl}$ with TPPA reactivity in the CSF; (3) other conditions that could cause neurological symptoms, such as myelopathy, were ruled out, as suggested by the European guideline on the management of syphilis [11]. Patients with NS were dichotomized into asymptomatic neurosyphilis (ANS) and symptomatic neurosyphilis (SNS) groups according to whether they had clinical complaints or not, with the ANS patients being discovered either in a premarital physical examination or accidentally [7].

\section{Imaging Data}

The neuroradiological conclusions were retrospectively reviewed for all patients with NS, including $\mathrm{CT}$ and MR imaging, that had been obtained by independent radiologists, and these were checked by other experienced radiologists who were unaware of the clinical diagnoses. Neuroimaging data included both the baseline examination and follow-up re-examinations. The detailed imaging parameters were as follows: cranial CT was performed on a 16 multidetector row CT (Brilliance, Philips, the Netherlands), and MRI was acquired on a 1.5-T or 3.0-T MRI system (Magnetom avanto $1.5 \mathrm{~T}$, Siemens, Berlin, Germany; Signa hdxt 3.0 T, GE Healthcare, Milwaukee, WI, USA). The MRI studies included diffusion weighted imaging, and T1-weighted spin echo sequences, T2weighted, and fluid attenuated inversion recovery imaging in different planes before and after intravenous injection of gadolinium-based contrast agents.

\section{Clinical Data}

The data extracted included age, sex, skin presentation, coexisting conditions (diabetes mellitus, high blood pressure, hypercholesterolemia, coronary artery disease, chronic nephritis and other infectious diseases), CSF testing results, first-visit department, and therapeutic medication. Neurological presentations and outcomes at baseline and follow-up were also reviewed.

\section{Statistical Analysis}

Results are reported as the mean \pm standard deviation (age and CSF protein), median with minimum and maximum values or interquartile range (quantitative variables), or frequencies and percentages (categorical variables). Independent sample $t$-tests or non-parametric Mann-Whitney $U$ tests were employed to determine the differences between SNS and ANS in terms of the quantitative variables. The chisquare test or Fisher's exact test were used for between-group comparisons of categorical variables. Differences of $P<0.05$ were considered significant. Statistical analyses were performed using SPSS software (version 19.0; IBM Corp. Armonk, NY, USA). 


\section{RESULTS}

As shown in Fig. 1 and Table 1, 102 patients with NS who were hospitalized during the study period were retrieved from the hospital information system. These patients included 60 cases $(58.8 \%)$ of SNS and 42 cases (41.2\%) of ANS. Univariate analysis showed statistically significant differences in the clinical presentations between the ANS and SNS patient groups, except for CSF white blood cells $(P=0.079)$ and the imaging characteristics of cerebral infarction and demyelination (both $P>0.05$ ). A total of 92 patients received anti-syphilitic treatment, patients with SNS had substantial improvement of clinical symptoms after treatment, and CSF re-examination in patients with ANS showed improvement in biochemical indicators, including decreased levels of white blood cells and proteins.

The 60 cases of SNS showed a wide variety of abnormal symptoms and signs, including psychological and behavioral disorders (18.3\%, $11 / 60)$, headache and dizziness $(26.7 \%, 16 / 60)$, cognitive decline $(10.0 \%, 6 / 60)$, sensory disturbance $(8.3 \%, 5 / 60)$, seizure or dysarthria (both

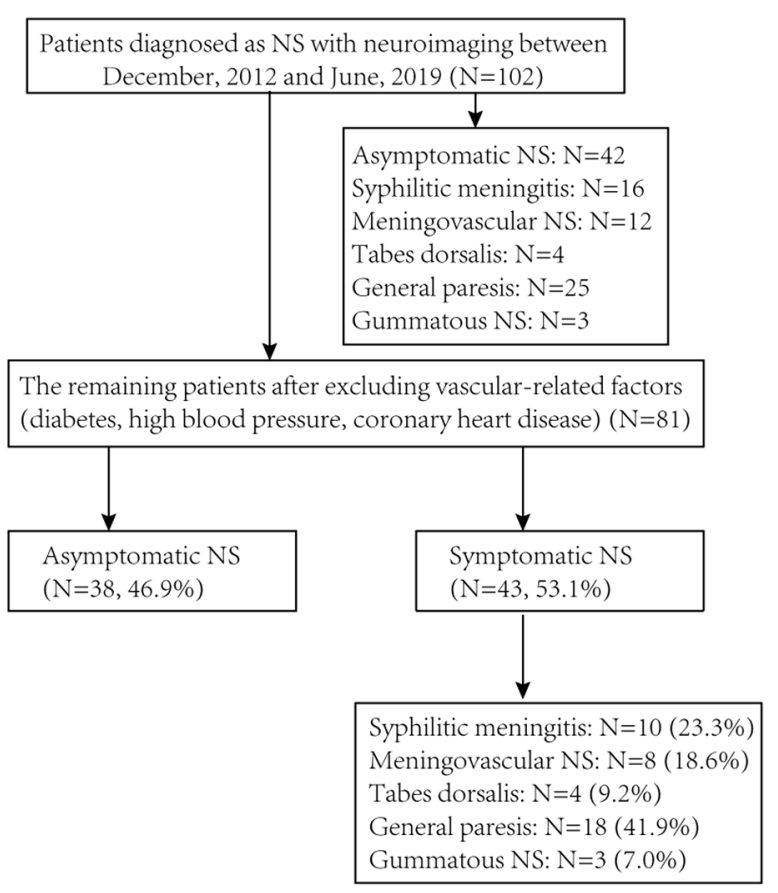

Fig. 1 Flow chart for screening patients with neurosyphilis
$6.7 \%, 4 / 60)$, weakness of limbs or hypopsia (both $5.0 \%, 3 / 60$ ), urinary or rectal incontinence $(3.3 \%, 2 / 60)$, aphasia or affective disorders $(3.3 \%, 2 / 60)$, and ataxia or oculomotor paralysis (both $1.7 \%, 1 / 60$ ). Notably, the patients with NS also manifested with a variety of imaging characteristics, including cerebral infarction or hemorrhage, atrophy, demyelination, arteritis, encephalitis, and hippocampal sclerosis (Table 1). Among the 81 patients without other coexisting diseases, 19 patients underwent MRI re-examinations (29 in total) during the follow-up period, with these including 4 cases of ANS, 4 cases of meningovascular NS, 8 cases of general paresis and 3 cases of gummatous NS. The median follow-up period was 10 months (interquartile range 5-16.5 months). All of the 19 patients who underwent MRI follow-up imaging received standardized anti-syphilitic therapy, 17/19 with high-dose intravenous penicillin, one with ceftriaxone, and one with doxycycline because of allergy to penicillin. No recurrence or worsening of their clinical symptoms (such as dizziness, headache, cognitive decline, visual or speech impairment) was detected at discharge or during follow-up; some of them are detailed in Table 2.

Comparisons between follow-up and baseline imaging showed that some patients had imaging progression despite being treated with standardized anti-syphilitic therapy (Table 3). First, infarction was a common manifestation of NS, with more than half of the patients $(52.6 \%$, 10/19; 3 with ANS and 7 with SNS) presenting with new infarction lesions after treatment. These lesions were of lacunar or acute type, and were partially dispersed in the cortex and subcortex, basal ganglia, cerebellar and brainstem areas. Second, most of the patients $(68.4 \%$, 13/19) initially showed cerebral atrophy, with all eight cases of general paresis showing brain atrophy. The six cases of NS that did not show brain atrophy were in patients with asymptomatic $(n=2)$, meningovascular $(n=1)$, or gummatous NS $(n=3)$. Notably, two patients with ANS showed brain atrophy, despite there being no obvious clinical symptoms. Moreover, 9/19 patients showed more severe brain atrophy after treatment. The initial MR imaging showed 


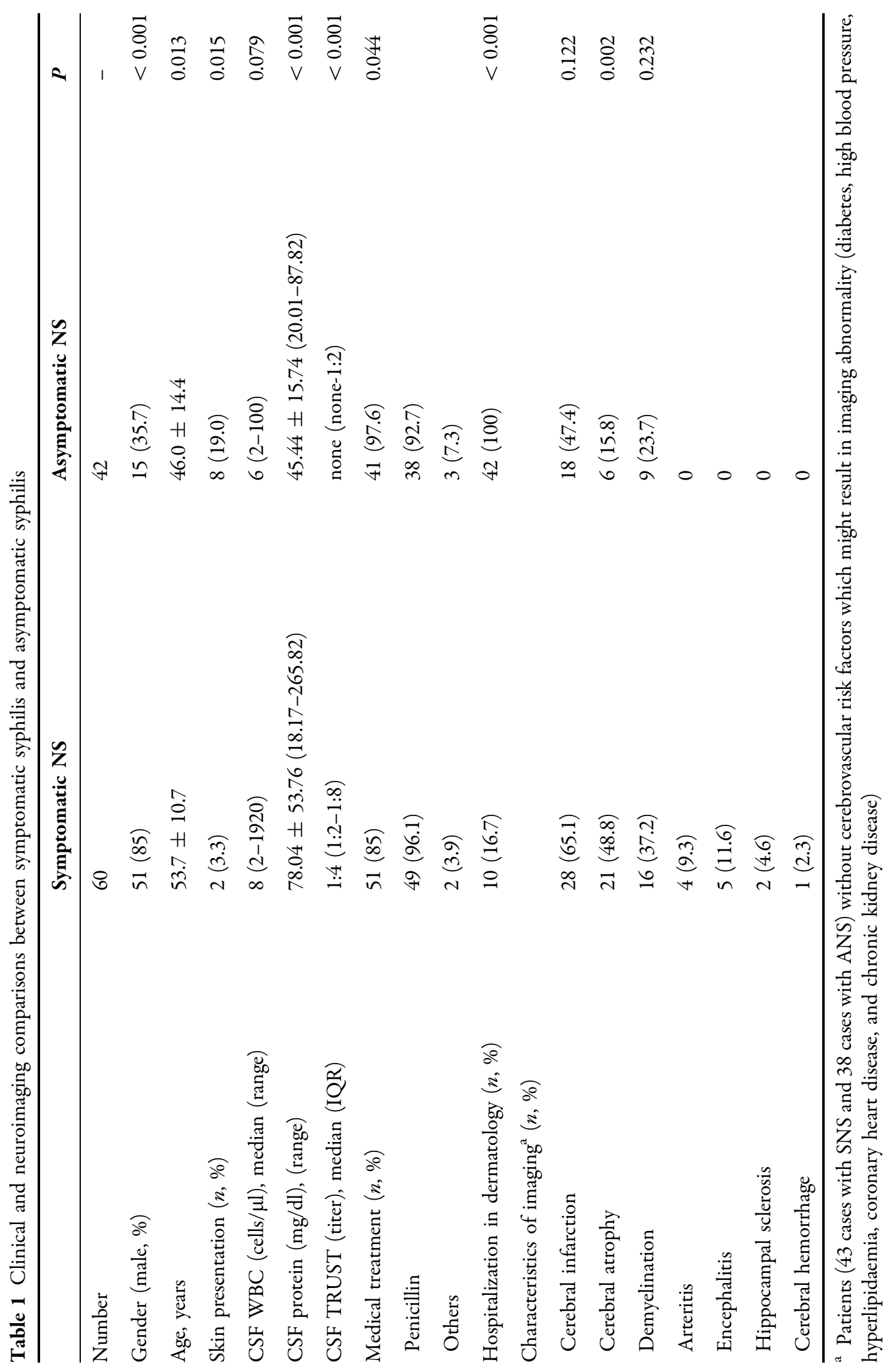




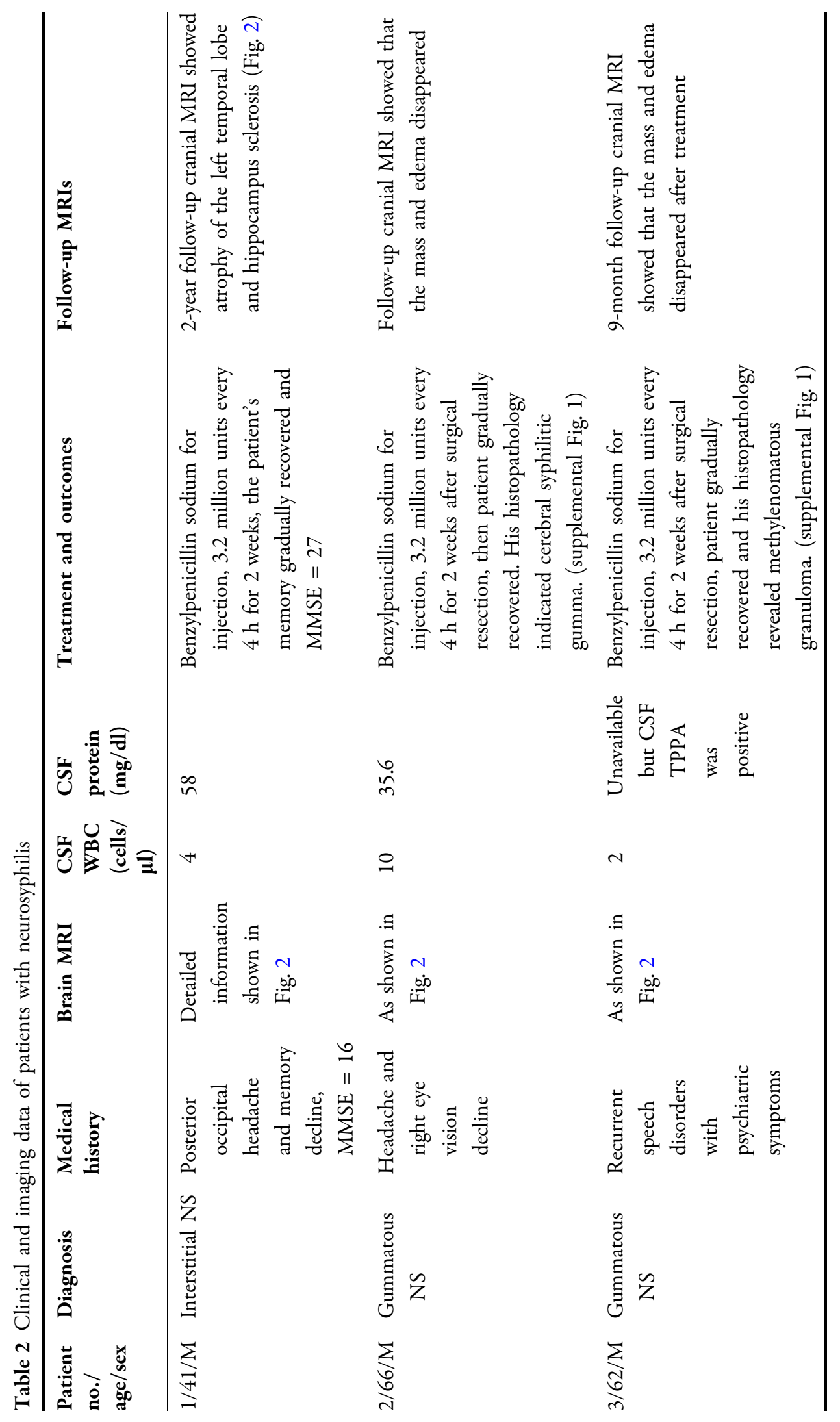




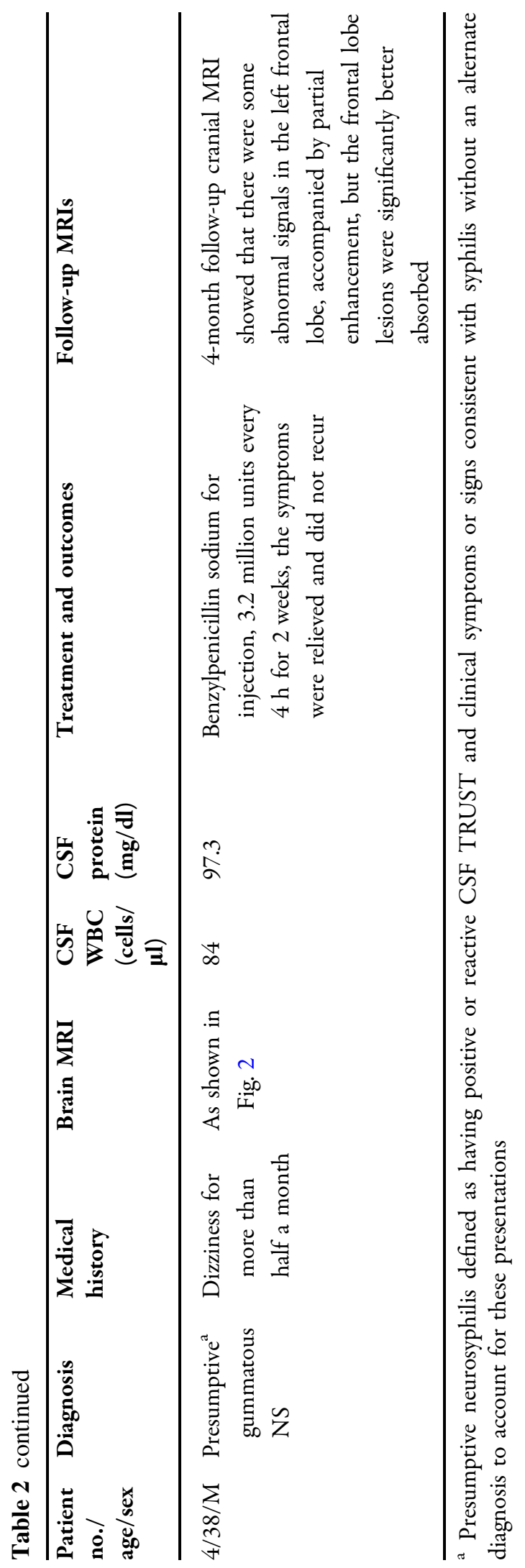

white matter hyperintensities in more than half of the patients $(57.9 \%, 11 / 19)$, with these lesions progressing during follow-up in three patients. Two of these patients with meningovascular NS showed increased basal ganglia and brainstem lesions, and the other patient with general paresis showed increased basal ganglia, brainstem and subcortical lesions. Additionally, hippocampal sclerosis $(n=1)$ resulting from a secondary change in intracranial inflammation also occurred, as shown in Table 2.

\section{DISCUSSION}

NS is commonly known as "the great mimicker" because of its wide range of clinical symptoms, and although it has classically been divided into early and late forms, there are some overlaps in clinical features between them. In our cohort, $41.2 \%(42 / 102)$ of NS patients had no clinical presentations, and it was therefore difficult to make a diagnosis at their first visit. Importantly, both ANS and SNS patients may initially show multiple neuroimaging abnormalities, and we found a large overlap in neuroimaging features between them. Although the use of anti-syphilitic treatment reduced the incidence of disease progression in the 19 patients with follow-up neuroimaging, this imaging indicated that some changes might be irreversible or even progressive.

The clinical symptoms of the NS patients were not specific, and there was a lack of accurate medical history and skin manifestations (possibly due to the patients' concealment of contact history and inattention to skin manifestations) [12], which could cause the patients with SNS to be sent to departments other than dermatology, making it difficult to achieve a timely and accurate diagnosis and appropriate treatment [5]. This study found that only $16.7 \%$ of SNS patients were admitted to the dermatology department, and that $15 \%$ of patients did not receive prompt antibiotic treatment. Moreover, the mean age of SNS patients was higher than that of the ANS patients, which might have been related to the course of the disease, because the clinical symptoms change over time. Previous literature has reported that NS 


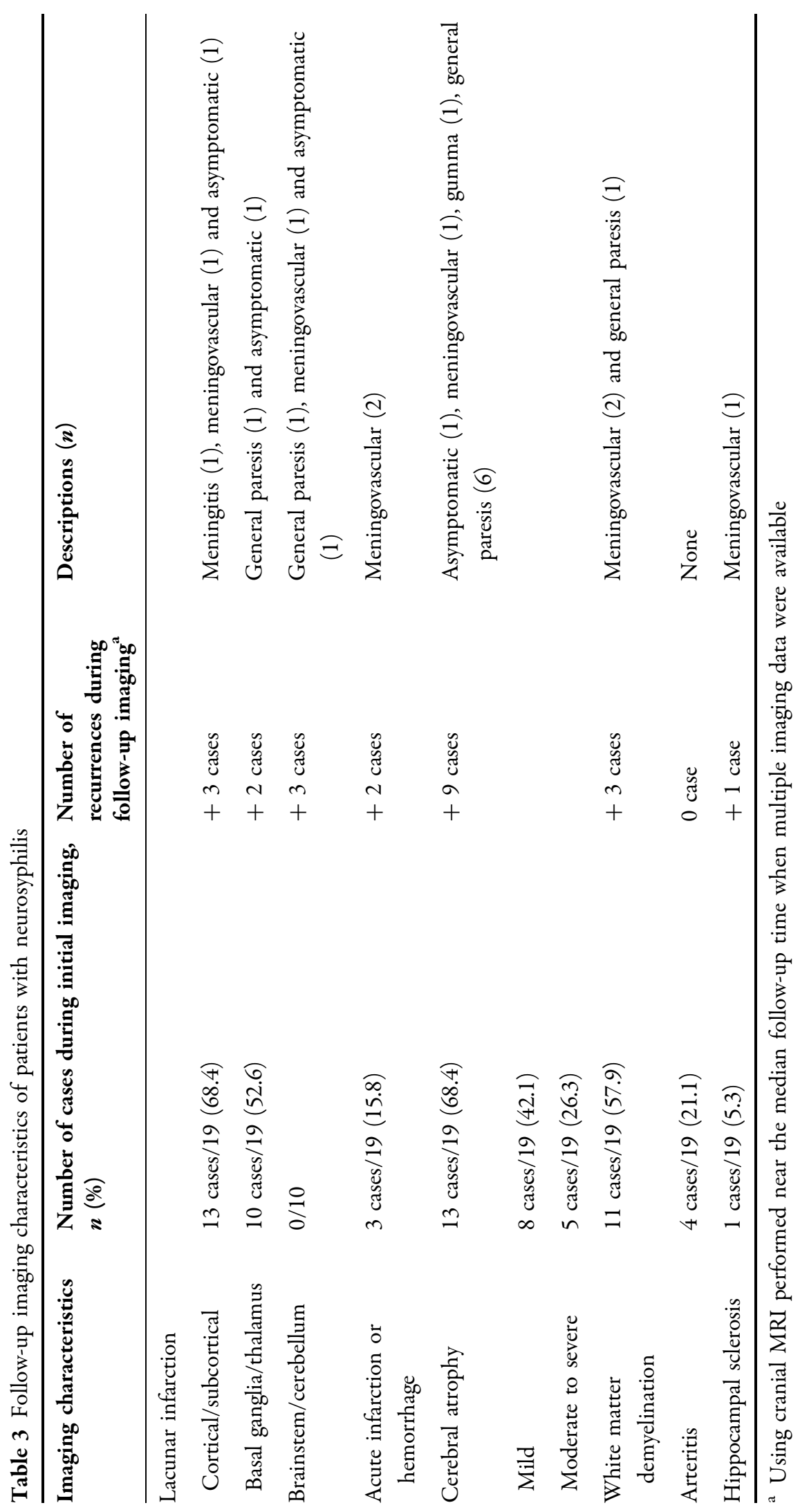



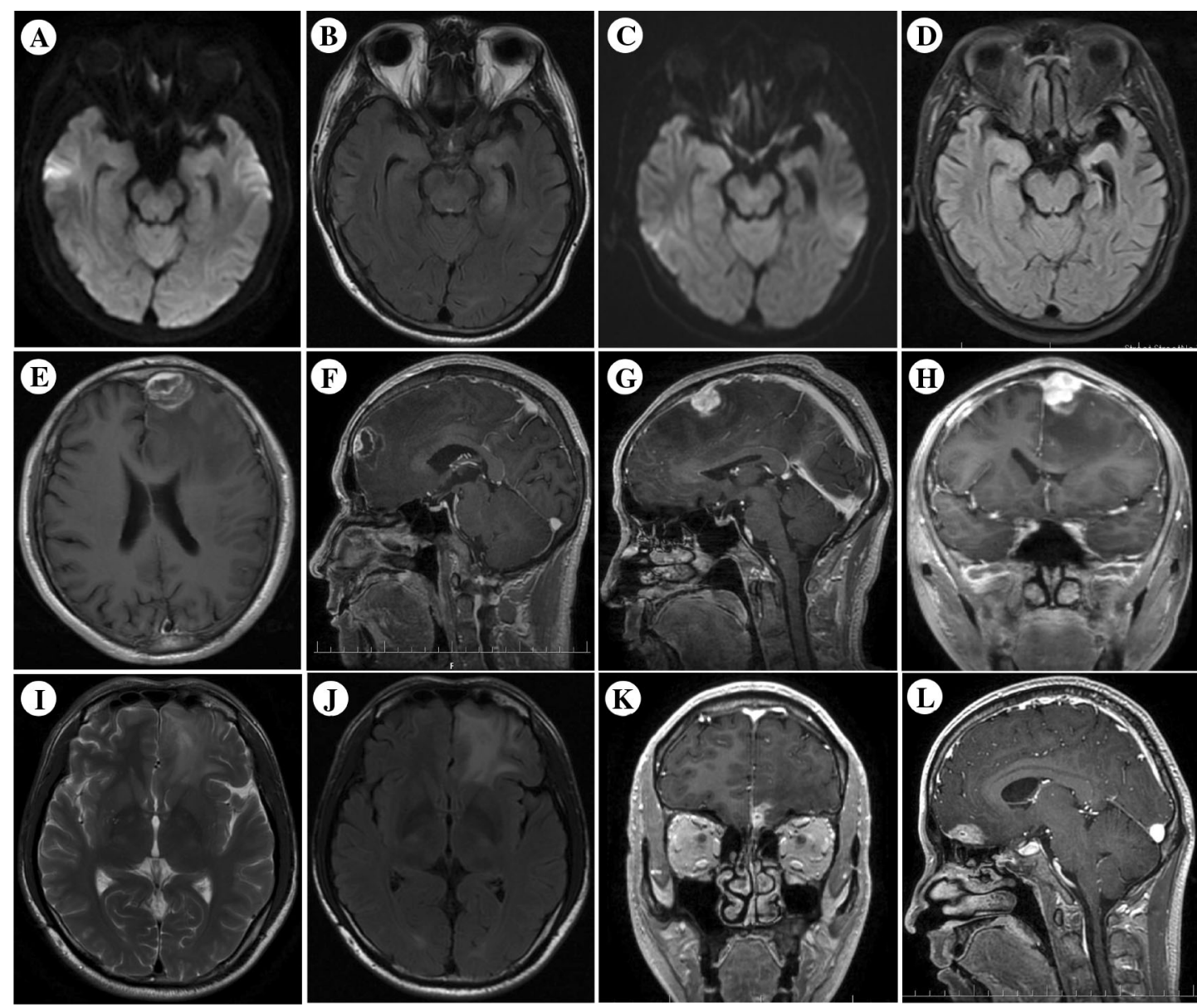

Fig. 2 Neuroimaging of NS patients. Case 1 patient's initial MRI shows lesions with abnormal signal in the left temporal lobe and hippocampus, suggesting acute inflammatory impairment. Two years after anti-syphilitic treatment, follow-up cranial MRI shows atrophy of the left temporal lobe and ipsilateral sclerosis of the hippocampus (a, c: DWI; b, d: FLAIR images). Case 2 patient's contrastenhanced T1-weighted MR image shows nodular enhanced lesion in the left frontal lobe, surrounding area

is low-intensity edema (e, f). Case 3 patient's contrastenhanced T1-weighted MR image shows nodular enhanced lesion in the left frontal lobe, surrounding area is low-intensity edema $(\mathbf{g}, \mathbf{h})$. Case 4 patient's T2-weighted and Flair MR images show cortical nodular high-intensity lesion at the bottom of frontal lobe $(\mathbf{i}, \mathbf{j})$, and his contrastenhanced T1-weighted MR images show nodular enhanced lesions $(\mathbf{k}, \mathbf{l})$

can occur during any stage of the syphilitic infection [13], even if the patient does not show obvious clinical symptoms; in such cases, CSF syphilis serological testing can assist in the diagnosis.

NS lacks specific imaging changes, and can show similar imaging characteristics at different stages of the disease, as shown in our study and others [14]. Possible explanations for the imaging-related phenomena may include the following. For NS to be recognized as subacute or

chronic CNS inflammation, the organism first needs to break through the blood-brain barrier, and the pathology of diffuse inflammatory activity may include meningitis, vasculitis, and even vasculitis-related infarct or hemorrhage, mainly involving the medium to large vessels and their perforating arteries. Consequently, NS patients frequently show abnormal neuroimaging findings of infarcts in the basal ganglia, brainstem, and cortical and subcortical regions, as in our patients [15-17]. Brain atrophy reflects 
a decrease in the volume of brain parenchymal tissue and usually affects the limbic lobe, temporal lobe, and hippocampus. It is the result of impaired brain tissue metabolism and function, which finally results in the death of neurons, glial hyperplasia, and cognitive decline $[9,17,18]$. Even when patients present with no neurological symptoms or signs, abnormal imaging findings may still be detected because of the involvement of the small vessels feeding deep and subcortical white matter, and ischemia emerging as a consequence of the syphilitic infection [18]. Thus, a diagnosis of NS cannot be excluded for patients showing the presence of clinically unexplained imaging abnormalities such as brain infarction or atrophy.

Like the diverse neuroimaging findings, the etiology underlying neuroimaging progression is also complicated. According to the stage of syphilis, most of the patients in this study were limited to latent and tertiary syphilis. Neurological findings in tertiary syphilis are related to a systemic hypersensitivity reaction to the microorganism or its remnants within the brain tissue. Neurological manifestations could be improved despite standardized treatment, but the immunological reaction might continue after treatment, albeit at a slower pace, resulting in neuroimaging progression $[19,20]$. In addition, use of antibiotics other than penicillin and short-term follow-up could also be responsible for progression. As for the detailed etiologies and pathological mechanism of disease progression, further research is needed.

This study is subject to a number of limitations. It is a single-center, retrospective study with a low sample size, which might decrease the explanatory power. The patients with SNS were also not stratified according to the stage of NS, and the progression of neuroimaging findings might differ between different stages. In addition, most patients had no available data on further treatment and long-term follow-up, which is also one of the difficulties in the clinical treatment of NS. This lack of data meant that correlation analysis between CSF serological results and neuroimaging features could not be performed: analysis that is worthy of further study with more samples.

\section{CONCLUSION}

NS has a wide range of clinical presentations and shows various neuroimaging features, and there are several overlaps in the neuroimaging manifestations between ANS and SNS patients. Additionally, their follow-up neuroimaging may show progression even with standardized treatment.

\section{ACKNOWLEDGEMENTS}

We thank the department of pathology and medical imaging of our hospital for their work on data acquisition.

Funding. This study was supported by a scientific research project of Wannan Medical College (Grant no. WK2019ZF01, Wuhu, China). The journal's Rapid Service Fee was funded by the authors.

Medical Writing Assistance. We thank Karl Embleton, Ph.D., from Liwen Bianji, Edanz Group China (www.liwenbianji.cn/ac), for editing the English text of a draft of this manuscript. The support was funded by Wannan Medical College.

Authorship. All named authors meet the International Committee of Medical Journal Editors (ICMJE) criteria for authorship for this article, take responsibility for the integrity of the work as a whole, and have given their approval for this version to be published.

Authorship Contributions. Cai-Feng He and Xian-Jin Shang contributed equally to the work and are co-first authors.

Disclosures. Xian-Jin Shang, Cai-Feng He, Biao Tang, Xiao-Li Chang, Chao Ci and Hong Sang have nothing to disclose.

Compliance with Ethics Guidelines. The study protocol was approved by Wannan Medical College (IRB: 201201) with a waiver for informed consent from participants because of its retrospective nature. 
Data Availability. The datasets generated during and/or analyzed during the current study are not publicly available but are available from the corresponding author on reasonable request.

Open Access. This article is licensed under a Creative Commons Attribution-NonCommercial 4.0 International License, which permits any non-commercial use, sharing, adaptation, distribution and reproduction in any medium or format, as long as you give appropriate credit to the original author(s) and the source, provide a link to the Creative Commons licence, and indicate if changes were made. The images or other third party material in this article are included in the article's Creative Commons licence, unless indicated otherwise in a credit line to the material. If material is not included in the article's Creative Commons licence and your intended use is not permitted by statutory regulation or exceeds the permitted use, you will need to obtain permission directly from the copyright holder. To view a copy of this licence, visit http://creativecommons.org/licenses/by$\mathrm{nc} / 4.0 /$.

\section{REFERENCES}

1. Ropper AH. Neurosyphilis. N Engl J Med. 2019;381: 1358-63.

2. Ghanem KG. REVIEW: neurosyphilis: a historical perspective and review. CNS Neurosci Ther. 2010;16:e157-68.

3. Hook EW 3rd. Syphilis. Lancet. 2017;389:1550-7.

4. Janier M, Hegyi V, Dupin N, et al. 2014 European guideline on the management of syphilis. J Eur Acad Dermatol Venereol. 2014;28:1581-93.

5. Zhang HL, Lin LR, Liu GL, et al. Clinical spectrum of neurosyphilis among HIV-negative patients in the modern era. Dermatology. 2013;226:148-56.

6. Liu H, Zhao ZB, You NX. Diversity in clinical manifestations and imaging features of neurosyphilis: obstacles to the diagnosis and treatment (report of three cases). Int J Neurosci. 2018;128:785-90.

7. Li W, Jiang $\mathrm{M}, \mathrm{Xu} \mathrm{D}$, et al. Clinical and laboratory characteristics of symptomatic and asymptomatic neurosyphilis in HIV-negative patients: a retrospective study of 264 cases. Biomed Res Int. 2019;2019:2426313.

8. Stefani A, Riello M, Rossini F, et al. Neurosyphilis manifesting with rapidly progressive dementia: report of three cases. Neurol Sci. 2013;34:2027-30.

9. Wang X, Yang Y, Wang X, Li C. MRI findings and early diagnosis of general paresis of the insane. Neurol Res. 2014;36:137-42.

10. Kodama K, Okada S, Komatsu N, et al. Relationship between MRI findings and prognosis for patients with general paresis. J Neuropsychiatry Clin Neurosci. 2000;12:246-50.

11. French P, Gomberg M, Janier M, et al. IUSTI: 2008 European Guidelines on the management of syphilis. Int J STD AIDS. 2009;20:300-9.

12. Drago F, Merlo G, Ciccarese G, et al. Changes in neurosyphilis presentation: a survey on 286 patients. J Eur Acad Dermatol Venereol. 2016;30:1886-900.

13. Marra C, Sahi S, Tantalo L, et al. Enhanced molecular typing of treponema pallidum: geographical distribution of strain types and association with neurosyphilis. J Infect Dis. 2010;202:1380-8.

14. Czarnowska-Cubala M. Neurosyphilis and brain magnetic resonance imaging. Int J Dermatol. 2015;54:863.

15. Holland BA, Perrett LV, Mills CM. Meningovascular syphilis: CT and MR findings. Radiology. 1986;158: 439-42.

16. Khamaysi Z, Bergman R, Telman G, Goldsher D. Clinical and imaging findings in patients with neurosyphilis: a study of a cohort and review of the literature. Int J Dermatol. 2014;53:812-9.

17. Peng F, Hu X, Zhong X, et al. CT and MR findings in HIV-negative neurosyphilis. Eur J Radiol. 2008;66: $1-6$.

18. Brightbill TC, Ihmeidan IH, Post MJ, et al. Neurosyphilis in HIV-positive and HIV-negative patients: neuroimaging findings. AJNR Am J Neuroradiol. 1995;16:703-11.

19. Hens MJ, Lolli F, Martín-Moro M, Giménez-Roldán S, Link H. High-dose intravenous-penicillin in neurosyphilis: effect on intrathecal synthesis of IgG, IgM, IgA and IgD. Acta Neurol Scand. 1990;82:381-5.

20. Pedersen NS, Kam-Hansen S, Link H, Mavra M. Specificity of immunoglobulins synthesized within the central nervous system in neurosyphilis. Acta Pathol Microbiol Immunol Scand C. 1982;90: 97-104. 